\title{
Factores del capital humano y desempeño en instituciones de educación media superior
}

\author{
América Lorena GonzÁlez Cisneros* \\ Norma Angélica Pedraza Melo**
}

La presente investigación muestra la relación existente entre el capital

Palabras clave

humano, componente del capital intelectual, y el desempeño educativo en el sector público. Se integró una muestra de 178 individuos, docentes y administrativos de cuatro instituciones de educación media superior. La confiabilidad del instrumento se determinó a través del alfa de Cronbach. En la determinación de la estructura factorial se empleó el análisis factorial exploratorio (AFE) y se utilizaron herramientas de estadística descriptiva e inferencial (análisis de regresión múltiple, prueba $t$-student, ANOVA de un factor). Se identificaron tres factores del capital humano y un solo factor para desempeño educativo. Se determinó que el aprendizaje y colaboración es el factor del capital humano que contribuye a explicar el desempeño en los planteles educativos analizados. Esto representa implicaciones para la gerencia educativa en el diseño de políticas y programas de gestión del talento humano.

The present research shows the relationship between human capital, a component of intellectual capital, and educational performance in the public sector. In order to do this, we surveyed a sample of 178 individuals between teachers and administrators from four upper secondary education institutions. The reliability of the instrument was determined through Cronbach's Alpha coefficient. In order to determine the factorial structure, we used exploratory factor analysis (EFA) as well as descriptive and inferential statistics tools (multiple regression analysis, Student's $t$-test, one-way ANOVA). Three human capital factors and a single factor for educational performance were identified. It was determined that learning and collaboration are a human capital factor that contributes to explain the performance levels in the surveyed educational establishments. This has specific implications for educational management in the design of human talent management policies and programs.

Capital humano

Desempeño educativo

Institución pública

Educación media superior

Competencia del capital humano

Keywords

Human capital

Educational performance

Public institution

Upper secondary

education

Human capital

competence

Recepción: 16 de mayo de 2019 | Aceptación: 1 de febrero de 2021

DOI: https://doi.org/10.22201/iisue.24486167e.2021.174.59393

* Estudiante de Doctorado en Ciencias Administrativas de la Universidad Autónoma de Tamaulipas (México). Maestra en Administración. Líneas de investigación: capital humano y prácticas de recursos humanos; capital intelectual y desempeño en organizaciones públicas y privadas. Publicación reciente: (2019, en coautoría con N. Pedraza), "El desarrollo sustentable y su relación con la educación media superior en México", en Yesenia Sánchez y Mariana Zerón (eds.), Nuevas perspectivas del emprendimiento en México, México, Colofón. CE: america.gonzalez@uat.edu.mx. ORCID: https://orcid.org/0000-0003-3529-5729.

** Profesora investigadora y coordinadora del área de planeación y operación de proyectos en el Centro de Innovación y Transferencia del Conocimiento (CINOTAM) de la Universidad Autónoma de Tamaulipas (UAT) (México). Doctora en Administración. Líneas de investigación: capital humano y prácticas de recursos humanos; capital intelectual y desempeño en organizaciones públicas y privadas. Publicación reciente: (2021, en coautoría con J. Lavín y M. Álvarez, "Relación del capital intelectual y el desempeño en la administración pública: el caso de una auditoría estatal”, Cuadernos de Administración, núm. 34, pp. 1-20, en: https://revistas.javeriana.edu.co/index.php/ cuadernos_admon/article/view/28976.CE: napedraza@docentes.uat.edu.mx. ORCID: http://orcid.org/0000-00019566-2880 


\section{INTRODUCCIÓN}

El sector público cuenta con necesidades y objetivos específicos, distintos de los del sector empresarial, y actualmente se encuentra obligado a implementar procesos de asignación de recursos a través de estándares de desempeño (Leitner, 2004; OCDE 2018; Pérez, 2016); además, el desarrollo económico y político también se encuentra directa y fuertemente relacionado con la dinámica del ámbito educativo (Cabrol y Székely, 2012; Rojas et al., 2000).

Aun cuando las instituciones educativas del sector público no tienen como principal objetivo la obtención de recursos, se encuentran interesadas en medir su desempeño, como parte de un proceso de generación de valor (González-Loureiro y Moreira, 2011; OCDE, 2017). Para la presente sociedad del conocimiento, la educación es una variable determinante que influye directamente en la evolución y desarrollo de las naciones, y se considera como la principal influencia en su competitividad (López, 2016; Pérez, 2016). Concebir a la institución educativa solamente como un medio que facilita un servicio quedó en el pasado; ahora es considerada como elemento indispensable para mejorar el desempeño de las naciones (Arechavala y Sánchez, 2017; Broucker et al., 2017; Dal Molin et al., 2017; Orozco, 2014; Leišyte, 2016) al mismo tiempo que se reconoce la influencia de los factores externos (económicos, culturales y sociales) en su desempeño (Mallama, 2019). En este sentido, actualmente los organismos públicos educativos están más atentos y son más responsables del uso y aplicación de los recursos, además de que están obligados a demostrar su valía (Agasisti et al., 2016; OCDE, 2017); ello explica la popularización de diversos modelos de evaluación educativa orientados a medir aspectos como el plan de estudios, la plantilla docente, la infraestructura y la tecnología educativa, entre otros (Gómez y Valdés, 2019).

Hoy, en América Latina, existe un mayor interés en mejorar la calidad educativa y el desem- peño de los actores del sector (Cóndor y Remache, 2019; Fullan, 2019). En este sentido, uno de los principios básicos para la Organización de las Naciones Unidas para la Educación, la Ciencia y la Cultura (UNESCO) es proveer educación para todos a lo largo de toda la vida; sin embargo, dentro de los planteles educativos ha sido difícil establecer una adecuada gestión estratégica en donde se desarrollen los recursos humanos, los procesos de innovación y la generación del conocimiento (Fullan, 2019; Flores, 2020; Guzmán et al., 2020; Pérez y Pino, 2018; Segatto y Abrucio, 2017). De la mano con la llamada "nueva gestión pública", en los últimos años se ha buscado mejorar la administración educativa (Agasisti et al., 2016; Bueno et al., 2008; Kelly, 2004; Leitner, 2004; Quispe, 2020; Sánchez et al., 2009; Vidal, 2017; Wu et al., 2012), la cual no se distingue significativamente de la administración en el ámbito privado; la gerencia educativa podría concebirse como un proceso sinérgico coordinado por medio de funciones directivas como planear, organizar, dirigir y controlar (Chawla y Lenka, 2015; Morgado et al., 2019; Rondón, 2016).

Por otro lado, actualmente los esquemas de Estado relacionados con la eficiencia y eficacia escolar se han visto mermados; es ahí donde el capital intelectual, como perspectiva teórica, otorga valor (Chatterji y Kiran, 2017; Kelly, 2004; Ordoñez et al., 2020). La medición es importante cuando se requiere un sistema educativo capaz de evolucionar a través de la gestión del conocimiento (Agasisti et al., 2016; Bueno et al., 2008; Fazlagic, 2005; Kelly, 2004; Leitner, 2004; Sánchez et al., 2009; Segatto y Abrucio, 2017; Vidal, 2017; Wu et al., 2012); por ende, una institución educativa es efectiva en la medida en que su contexto interno y externo coadyuvan a alcanzar metas más altas de las planteadas originalmente; es decir, cuando una escuela aporta valor, cuando es competitiva (Cabrol y Székely, 2012; OCDE, 2017, 2018; Quispe, 2020; Rowe et al., 1995).

Las investigaciones empíricas de temas relacionados con el capital intelectual y la 
educación son escasas (Allui y Sahni, 2016; Ayaz et al., 2018; Barreto y Azeglio, 2013; González-Loureiro y Moreira, 2011). Bahrami et al. (2013) indican que la generación y difusión de los conocimientos es el principal objetivo de las instituciones educativas, ya que permiten el establecimiento de saberes, experiencias, valores, información, percepciones e ideas, entre otras (Toalá-Sánchez et al., 2017), y consideran a la investigación y a los recursos humanos como los elementos más importantes, aun cuando su desarrollo, medición y evaluación han sido limitados (Sánchez et al., 2009).

Para Bontis (2001), el interés por los intangibles - y específicamente por el capital intelectual-, es un nuevo sentido de crear valor organizacional, mismo que, en fechas recientes, se ha extendido de lo privado a lo público (Guthrie y Dumay, 2015; Sánchez et al., 2009). Para algunos investigadores el capital intelectual trata de enlazar las aspiraciones del sistema gubernamental a través de las metas y objetivos establecidos por las instituciones educativas, ya que con ello se estaría garantizando la generación de valor para el futuro, y la alineación con las políticas de Estado (Bueno et al., 2008; Chatterji y Kiran, 2017; Kelly, 2004; Vidal, 2017).

Ramirez y Gordillo (2014) conceptualizan al capital intelectual educativo como los activos no físicos de la institución: procesos, patentes, innovaciones, conocimiento tácito de los miembros, reconocimiento de la sociedad, talentos, habilidades, redes de colaboradores, contactos, entre otros (Castro, 2015; Rondón, 2016; Toalá-Sánchez et al., 2017). Las universidades son el campo de estudio ideal de estos temas. Hace algunos años, las instituciones educativas europeas transitaron por un camino de transformación con la intención de lograr transparencia y flexibilidad y así volverse más competitivas (Sánchez et al., 2009).

En la última década se ha buscado desarrollar métodos para medir los intangibles (Sánchez et al., 2009), pero desafortunadamente su medición no se ha implementado de manera suficiente en las instituciones educativas y sus afines (González-Loureiro y Moreira, 2011; Segatto y Abrucio, 2017; Vidal, 2017; Wu et al., 2012); sin lugar a dudas, hacen falta modelos holísticos con visión gerencial que brinden bases sólidas para la consecución de dichas investigaciones en el ámbito educativo.

Con base en lo anterior, la encomienda del presente estudio es validar la hipótesis de relación de dependencia entre los factores del capital humano y su contribución al desempeño educativo en instituciones de educación media superior. En México, este nivel escolar enfrenta retos significativos que van desde temas educativos como la generación de mayores oportunidades de ingreso, equidad, inclusión, no abandono escolar y una alineación adecuada de los egresados, educativa y laboral, hasta temas de gestión administrativa como la profesionalización y capacitación docente, la infraestructura y el equipamiento, entre otras (Cetzal et al., 2020).

Este estudio tiene los siguientes objetivos de investigación: i) analizar la relación entre las dimensiones del capital humano y el desempeño educativo; ii) contrastar la percepción del personal encuestado en relación con la valoración de los constructos de capital humano y el desempeño educativo, en función del género y tipo de puesto que ocupa en la institución; y iii) contrastar la percepción del personal encuestado en relación con la valoración de los constructos de capital humano y la competitividad educativa por plantel educativo.

Se realizó un análisis con enfoque cuantitativo, y para ello de aplicó un cuestionario al personal docente y administrativo de cuatro instituciones de educación media superior (EMS) en Ciudad Victoria, Tamaulipas (México), pertenecientes a la Unidad de Educación Media Superior Tecnológica Industrial y de Servicios (UEMSTIS). A partir de Bontis (1998), se aplicó un instrumento piloto que ha sido traducido y adaptado del modelo original de dicho autor. Esta investigación se realizó durante el mes de marzo de 2019. Se conformó 
una muestra no probabilística de 178 individuos entre personal docente y administrativo.

Este artículo se estructura en cinco apartados: el primero habla de la importancia estratégica del capital humano en el desempeño educativo; el segundo muestra evidencias empíricas relacionadas con el tema; en el tercero se muestra el diseño metodológico. Dentro de los resultados (cuarta sección) se presentan los principales hallazgos de esta investigación, para mostrar, en la última parte de este trabajo, las conclusiones, basadas en el análisis de la relación entre los factores del capital humano y la competitividad de las instituciones educativas analizadas.

\section{IMPORTANCIA ESTRATÉGICA}

\section{DEL CAPITAL HUMANO EN}

LA COMPETITIVIDAD DE LAS

\section{INSTITUCIONES EDUCATIVAS PÚBLICAS}

La clasificación comúnmente aceptada del capital intelectual está conformada por el capital humano, estructural y relacional (Bontis, 1998; Leitner, 2004; Morales et al., 2020; Ramírez et al., 2007; Sánchez et al., 2009); para muchos autores, el capital humano es al que se le asigna mayor relevancia. En el ámbito privado, los investigadores han definido el capital humano como el elemento más difícil de abordar; Bontis (2001) considera que representa un medio estratégico de cambio, e indica que, al integrar conceptos como el conocimiento y las habilidades, aporta valor, aun y cuando éstos no le pertenezcan del todo a la institución (Barbosa-Chacón et al., 2015).

Para otros autores, el capital humano se basa en las capacidades colectivas que se requieren para resolver problemas con el entorno; aporta experiencia, habilidad, conocimiento y genera valor a la organización (Edvinsson y Sullivan, 1996). Autores como Queiroz et al. (2005), Cheng (2015) y Kucharčíková et al. (2015) relacionan el concepto con la competencia de los empleados (aptitudes, conocimientos permanentes, habilidades y experiencias), lo que permite aumentar el rendimiento y la competitividad.

En las instituciones de educación el capital humano (personal administrativo, docente, el dedicado a la investigación, directivo y de servicios, entre otros) ha sido definido escasamente (Castro, 2015). Leitner (2004) lo considera como el recurso más valioso dentro de las instituciones educativas, ya que es a través de él que se genera el conocimiento y con cuya experiencia, capacidad y creatividad, entre otras, mejora el desempeño institucional (Hili et al., 2017; Morales et al., 2020). Ramírez et al. (2007) definen al capital humano educativo como una suma de conocimientos tangibles e intangibles que se generan a través de investigación formal e informal. Otros autores consideran que representa el conocimiento que poseen los estudiantes, investigadores, docentes y administrativos, y señalan que dichos conocimientos dejarían de formar parte de los activos de la institución una vez que el personal abandona la institución (Bahrami et al., 2013; Orozco, 2014; Farah y Abouzeid, 2017; Ramírez y Gordillo, 2014).

Adicionalmente, representa el capital interno de la institución (valores, competencias, cultura, políticas) en sinergia con su capital externo (reputación, imagen y relaciones institucionales) (Cheng, 2015). Marina (2015), citado por Mallama (2019), señala que el capital humano es el responsable principal en la generación de resultados escolares de éxito, lo que concuerda con Leiva-Guerrero y Vásquez (2019), quienes señalan que investigaciones recientes sostienen que el capital humano es el factor que mayoritariamente impacta en los planteles educativos (Morales et al., 2020).

El éxito de una organización de educación depende de que el capital humano sea capaz de adaptarse a la vorágine de cambios del entorno; una institución educativa demuestra su adaptación cuando los elementos que la integran logran asimilar los cambios eficientemente en sus estrategias (Morales et al., 2020; Toalá-Sánchez et al., 2017; Zlate y 
Enache, 2015). Las instituciones educativas están obligadas a observar y dar seguimiento a su contexto educativo (Barbosa-Chacón et al., 2015), además de realzar el valor del capital humano mejorando los conocimientos, habilidades, destrezas y actitudes de quienes las conforman (Cóndor y Remache, 2019).

Algunos autores consideran que las percepciones y compromiso del capital humano, específicamente de los maestros, influyen directamente en el aprendizaje de los alumnos; en fechas recientes, una investigación llevada a cabo en 2016 en Arabia Saudita determinó los grandes problemas que tienen las instituciones educativas de aquella nación en el tema de capital humano y concluye que es necesario realizar más investigaciones con variables moderadoras respecto al tema (Allui y Sahni, 2016). Así mismo, Webster y Watson (2002) demostraron una relación sólida entre el rendimiento de los estudiantes y el nivel de eficiencia de los docentes en instituciones educativas. Identificar y gestionar al capital humano en las organizaciones educativas permite tener claras sus fortalezas y debilidades, con lo cual se incrementa el conocimiento, se estimula la innovación, se mejora la toma de decisiones y se garantiza la mejora de su desempeño (Allui y Sahni, 2016; Ayaz et al., 2018; Barreto y Azeglio, 2013; González-Loureiro y Moreira, 2011; Toalá-Sánchez et al., 2017).

\section{Algunas evidencias empíricas}

Medir y administrar estratégicamente al capital intelectual es importante para impulsar el desempeño organizacional. Bontis y Serenko (2009) proponen uno de los modelos más empleados en las organizaciones para predecir el efecto de las políticas aplicadas al capital humano en el logro de los objetivos propuestos; esto implica tres dimensiones: humano, estructural y relacional. En la mayoría de los modelos propuestos se considera que el capital humano es el factor que crea al capital estructural y al relacional (Sveiby, 1997; Edvinsson y Malone 1999; Queiroz et al., 2005). Bontis (2001) realizó un estudio empírico en el que estableció modelos conceptuales de capital intelectual y analizó su impacto en las organizaciones; para ello utilizó el análisis confirmatorio y mínimos cuadrados parciales (PLS). Así mostró una relación significativa entre el capital intelectual y el desempeño organizacional.

Estudios en el sector privado vinculan los elementos del capital intelectual (humano, estructural y relacional) con la tasa de crecimiento de las pequeñas y medianas empresas (pymes); las investigaciones dan cuenta de que el capital humano es el que explica el crecimiento en las organizaciones de este tipo (González-Loureiro y Moreira, 2011). Dentro del sector público, sin embargo, existen pocos modelos respecto al tema; uno es el propuesto por Sánchez et al. (2009), el cual mide el capital intelectual a través de cinco variables: capital humano, organización interna, relaciones exteriores, calidad y transparencia. En el mismo estudio se menciona a los "pasivos intelectuales", definidos como la falta de capital intelectual en una institución pública. Adicionalmente, algunos autores agregan dos capitales más en relación con el sector público: el compromiso social y medioambiental, y la transparencia de gestión (Queiroz et al., 2005).

Pocos modelos empíricos dan soporte para realizar la medición y generación de resultados del capital intelectual en las instituciones educativas (Ramírez y Gordillo, 2014), de allí el interés e importancia de realizar estudios sobre este constructo. Por ejemplo, algunas investigaciones hablan del rendimiento educativo y lo relacionan con la eficacia escolar utilizando regresión múltiple (Hill y Rowe, 1996); también se han operacionalizado modelos tradicionales con variables explicativas de un solo nivel. La literatura indica que existen problemas metodológicos en investigaciones que miden la competitividad educativa, puesto que los datos con los que se cuenta son complejos y provienen de diversas fuentes: docentes, personal directivo, administrativo, de servicios, 
estudiantes y padres de familia, entre otros, además de que el giro de la institución educativa también cuenta como factor determinante. Por ello Nava-Rogel y Mercado-Salgado (2011) sugieren la aplicación de estudios longitudina- les a través de modelos multinivel para realizar estudios empíricos en el área.

La Tabla 1 muestra algunos estudios empíricos que miden de alguna manera al capital humano en instituciones educativas:

Tabla 1. Estudios empíricos que miden el capital humano y el desempeño en instituciones educativas a nivel internacional

\begin{tabular}{|c|c|c|c|c|}
\hline Estudio & Autor(es) & Objetivo & $\begin{array}{c}\text { Características del } \\
\text { estudio }\end{array}$ & Resultado(s) \\
\hline $\begin{array}{l}\text { Modelo de gestión } \\
\text { del talento humano } \\
\text { Venezuela }\end{array}$ & $\begin{array}{l}\text { Rondón } \\
\text { (2016) }\end{array}$ & $\begin{array}{l}\text { Fortalecer el trabajo } \\
\text { corporativo dentro } \\
\text { de las organizaciones } \\
\text { educativas }\end{array}$ & $\begin{array}{l}\text { Cuantitativo. } \\
\text { Se analizaron } 20 \\
\text { instituciones } \\
\text { ( } 431 \text { docentes y } 40 \\
\text { directivos) }\end{array}$ & $\begin{array}{l}\text { El talento humano } \\
\text { dentro de las institu- } \\
\text { ciones educativas se } \\
\text { fortalece al desarro- } \\
\text { llar las capacidades } \\
\text { del personal, su } \\
\text { compromiso y su } \\
\text { acción }\end{array}$ \\
\hline $\begin{array}{l}\text { Modelo de gestión } \\
\text { del conocimiento y } \\
\text { capital intelectual } \\
\text { (CI) para docentes } \\
\text { universitarios } \\
\text { Ecuador }\end{array}$ & $\begin{array}{l}\text { Toalá- Sánchez et al. } \\
\text { (2017) }\end{array}$ & $\begin{array}{l}\text { Incrementar la com- } \\
\text { petitividad del sector } \\
\text { educativo }\end{array}$ & $\begin{array}{l}\text { Se utilizó el modelo } \\
\text { Intelect, que mide } \\
\text { el CI en activida- } \\
\text { des docentes y de } \\
\text { gestión académica: } \\
\text { selecciona, mide y } \\
\text { cuantifica a través de } \\
\text { indicadores }\end{array}$ & $\begin{array}{l}\text { Cuando se identifica } \\
\text { administra y organi- } \\
\text { za el conocimiento a } \\
\text { través de las habilida- } \\
\text { des docentes, se logra } \\
\text { un mejor desempeño } \\
\text { en la institución } \\
\text { educativa }\end{array}$ \\
\hline $\begin{array}{l}\text { Evaluación del } \\
\text { capital intelectual } \\
\text { en instituciones } \\
\text { educativas } \\
\text { Irán }\end{array}$ & $\begin{array}{l}\text { Khalkhali et al. } \\
\text { (2012) }\end{array}$ & $\begin{array}{l}\text { Analizar el capital } \\
\text { intelectual a través } \\
\text { del análisis factorial } \\
\text { exploratorio }\end{array}$ & $\begin{array}{l}\text { Se analizó a } 500 \text { ex- } \\
\text { pertos y especialistas } \\
\text { en educación }\end{array}$ & $\begin{array}{l}\text { El capital humano, el } \\
\text { relacional y estruc- } \\
\text { tural, coadyuvan } \\
\text { significativamente en } \\
\text { la calidad y la mejora } \\
\text { continua }\end{array}$ \\
\hline $\begin{array}{l}\text { Análisis del capital } \\
\text { intelectual y el desem- } \\
\text { peño organizacional } \\
\text { en instituciones } \\
\text { educativas públicas y } \\
\text { privadas } \\
\text { Rumania }\end{array}$ & $\begin{array}{l}\text { Zlate y Enache } \\
(2015)\end{array}$ & $\begin{array}{l}\text { Determinar la rela- } \\
\text { ción existente entre } \\
\text { el capital intelectual } \\
\text { y el desempeño } \\
\text { organizacional }\end{array}$ & $\begin{array}{l}258 \text { trabajadores de } \\
\text { instituciones públi- } \\
\text { cas y privadas }\end{array}$ & $\begin{array}{l}\text { Se determina que el } \\
\text { capital humano es el } \\
\text { elemento clave en la } \\
\text { mejora del desem- } \\
\text { peño organizacional } \\
\text { en estas instituciones } \\
\text { educativas }\end{array}$ \\
\hline $\begin{array}{l}\text { Análisis del capital } \\
\text { intelectual en el } \\
\text { rendimiento de } \\
\text { la investigación } \\
\text { universitaria } \\
\text { Pakistán }\end{array}$ & $\begin{array}{l}\text { Awan y Saeed } \\
(2014)\end{array}$ & $\begin{array}{l}\text { Analizar el impacto } \\
\text { que tiene el capital } \\
\text { intelectual en el } \\
\text { rendimiento de la in- } \\
\text { vestigación universi- } \\
\text { taria en instituciones } \\
\text { educativas paquista- } \\
\text { níes comparándolo } \\
\text { en dos centros de } \\
\text { educación }\end{array}$ & $\begin{array}{l}\text { Utiliza un enfoque } \\
\text { exploratorio para } \\
\text { desarrollar el modelo } \\
\text { conceptual estudio } \\
\text { de caso en dos gran- } \\
\text { des universidades } \\
\text { del sector público. } \\
\text { Se utilizan datos } \\
\text { secundarios y la } \\
\text { correlación }\end{array}$ & $\begin{array}{l}\text { Los componentes del } \\
\text { capital intelectual } \\
\text { tienen un impacto } \\
\text { significativo en el } \\
\text { rendimiento de las } \\
\text { instituciones: el } \\
\text { capital humano se } \\
\text { encuentra en primer } \\
\text { lugar, seguido por el } \\
\text { capital estructural y } \\
\text { el relacional }\end{array}$ \\
\hline
\end{tabular}

Fuente: elaboración propia a partir de autores citados. 
Habrá que añadir, para complementar la información de la Tabla 1, que otros autores han realizado investigaciones, en su mayoría cuantitativas, en las que identifican relaciones significativas entre el capital humano y el desempeño de los planteles (Ayaz et al., 2018; Castro, 2015; Cuentas, 2018; González, 2018; Hili et al., 2017; Horn y Murillo, 2016; Lora et al., 2018; Morales et al., 2017; Morales et al., 2020; Siu, 2020; Villagra et al., 2018; Xu et al., 2011; Zlate y Enache, 2015). Por su parte, Martínez-Chairez y Guevara-Araiza (2015) y Perez et al. (2005) encontraron una relación negativa en sus investigaciones, al igual que Decker et al. (2004).

En México son pocos los estudios realizados que miden el capital intelectual - y en menor medida al capital humano- en las organizaciones de educación pública; por mencionar un ejemplo, existe evidencia de que el capital humano dedicado a la docencia influye principalmente sobre el capital humano dedicado a la investigación, mismo que, en menor intensidad, afecta al capital estructural educativo y, por último, al capital relacional (Nava-Rogel y Mercado-Salgado, 2011). Adicionalmente, la investigación realizada por Morales et al. (2020) a 81 directivos escolares de instituciones de educación básica al sur de Sonora, México, determinó que de los activos intangibles que forman parte del capital intelectual, el capital humano, a través de su formación y capacitación, es la variable de mayor importancia y contribución al momento de explicar el desempeño educativo en esas instituciones (58.1 por ciento).

Como resultado de la revisión de literatura se observa que existe más desarrollo y medición de activos intangibles dentro del sector privado, y que la producción académica en instituciones educativas del sector público sobre el tema es limitada (Guthrie y Dumay, 2015; Ramírez et al., 2007). Actualmente, no hay obligación o recomendaciones formales para que las instituciones educativas generen informes de su capital intelectual (Ramírez y Gordillo, 2014); mientras no se le dé relevancia al tema, difícilmente se logrará reconocer la importancia de este tipo de recursos intangibles en la gestión de las instituciones. Entonces, es necesario investigar, en relación con los temas gerenciales, qué es lo que más influye en estas instituciones y cómo se da; para diversos autores, el capital humano es el punto de partida (Barbosa-Chacón et al., 2015; Barreto y Azeglio, 2013; Farah y Abouzeid, 2017; Guzmán y Marín, 2011; González-Loureiro y Moreira, 2011; Martín et al., 2013).

Con base en la revisión de literatura, se generó un modelo teórico que se sometió a validación (Fig. 1) en la presente investigación, a fin de evaluar la hipótesis de que existe una relación positiva y significativa entre los factores que constituyen el capital humano y el desempeño educativo en instituciones de educación media superior (EMS). Esta fase constituye una etapa preliminar de un proyecto de investigación de mayor cobertura en el estudio de activos intangibles, como la gestión del conocimiento, el aprendizaje organizacional y el capital humano.

\section{MATERIAL Y MÉTODO}

\section{Características del estudio}

Este estudio se desarrolla desde el enfoque cuantitativo, con alcance explicativo, mediante un diseño transversal y no experimental. La recolección de los datos ocurrió en un periodo específico de tiempo (marzo de 2019), sin que se ejerciera ninguna influencia en los encuestados para responder las preguntas formuladas en el instrumento. Los datos recabados se analizaron a través de la estadística descriptiva e inferencial (Hernández-Sampieri y Mendoza, 2018; Creswell et al., 2013).

\section{Unidad de análisis, población y muestra}

El análisis de los datos se realizó en cuatro planteles de educación media superior (EMS) que corresponden al nivel de gestión de la federación y que se encuentran localizados en Ciudad Victoria, Tamaulipas (México). Este 
tipo de planteles educativos se conocen como Centros de Bachillerato Tecnológico, Industrial y de Servicios (CBTIS), y tienen la misión de formar capital humano con competencias técnicas en diversas especialidades. Por tratarse de la fase piloto del instrumento, y al representar este estudio una fase preliminar de una investigación de mayor alcance, sólo participaron cuatro centros $(271,236,119$ y 24).

La población objeto de estudio está constituida por personal docente $y$ administrativo activo en el periodo de análisis, el cual representaba una población total de 649 empleados de las cuatro instituciones que accedieron a colaborar. Cabe señalar que se corrió la invitación de responder el cuestionario a la totalidad de los empleados ( $\mathrm{N}=649)$, sin embargo, sólo devolvieron el instrumento 178 trabajadores, es decir que se obtuvo una tasa de respuesta de 27.42 por ciento; con estos cuestionarios se conformó finalmente una muestra no probabilística para esta investigación (Münch y Ángeles, 2009), de acuerdo con la distribución que se describe en la Tabla 2:

\section{Tabla 2. Cantidad de encuestados por plantel educativo}

\begin{tabular}{lc}
\hline Plantel & $\begin{array}{c}\text { Muestra no probabilís- } \\
\text { tica por plantel }\end{array}$ \\
\hline CBTIS 271 & 50 \\
\hline CBTIS 236 & 51 \\
\hline CBTIS 119 & 30 \\
\hline CBTIS 24 & 47 \\
\hline Total $(\mathrm{n})$ & 178 \\
\hline
\end{tabular}

Fuente: elaboración propia.

Este tamaño muestral $(\mathrm{n}=178)$ se estimó adecuado para proceder a la valoración del instrumento; se especifica que los cuestionarios fueron aplicados a través de un oficio de presentación emitido por la Coordinación Estatal de la UEMSTIS en el estado, el cual fue dirigido al personal directivo en cada uno de los cuatro planteles educativos a fin de que se coordinaran los trabajos para su aplicación.

\section{Técnica de recolección de datos}

Este estudio muestra resultados preliminares de la valoración del instrumento para el contexto de interés, puesto que el alcance del proyecto de investigación es analizar el fenómeno objeto de estudio en la totalidad de los planteles educativos de la UEMSTIS del estado de Tamaulipas. La escala utilizada para el diseño del instrumento de este estudio fue la propuesta por Bontis (1998), traducida y adaptada al contexto local (Pedraza et al., 2017).

El cuestionario quedó conformado por dos secciones (ver Anexo). La primera sección está integrada por 18 ítems, que son presentados al encuestado en escala tipo Likert de cinco puntos ( $1=$ nunca; $2=$ rara vez; $3=$ algunas veces; $4=$ la mayoría de las veces; $5=$ =siempre). Este apartado se enfoca a la recolección de datos de aspectos que caracterizan al capital humano, medidos desde la percepción que el personal docente y administrativo tiene de temas como el aprendizaje, la colaboración y el trabajo en equipo, entre otros (Bontis, 1998; Pedraza et al., 2017).

La segunda sección del instrumento fue dirigida a evaluar el desempeño de la institución educativa en los últimos tres años. Se desarrollaron 13 ítems, igualmente bajo escala tipo Likert de cinco puntos ( $1=$ pésimo; $2=$ malo; $3=$ regular; $4=$ bueno y $5=$ superior), para valorar aspectos como imagen institucional, innovación, calidad y desempeño. Adicionalmente se estableció una tercera sección para recabar datos generales del encuestado (puesto, antigüedad, edad, escolaridad, género), de plantel y zona geográfica.

\section{Técnicas de análisis de datos}

En el análisis de confiabilidad del cuestionario se determinó el alfa de Cronbach para los constructos de capital humano y desempeño; el criterio de aceptación establece la consistencia interna de un instrumento al obtener un 
valor de .70 como mínimo (Nunnally, 1995). Se aplicó la técnica del análisis factorial exploratorio (AFE) mediante la versión 23 del paquete estadístico SPSS (Statistical Package for the Social Sciences), a través del método de extracción de máxima verosimilitud y mediante rotación PROMAX (Hair et al., 2014). Dicha técnica multivariante es utilizada para determinar las relaciones en una cantidad amplia de variables, a fin de que la información pueda ser sintetizada en una menor cantidad de factores (Hair et al., 2014; Pérez y Medrano, 2010).

Se utilizó la prueba de esfericidad de Bartlett y la medida de adecuación muestral de Kaiser, Mayer-Olikin (KMO) para evaluar la conveniencia de aplicar el AFE a la matriz de correlaciones de datos. El criterio del índice de KMO muestra datos aceptables cuando se genera un valor igual o por arriba de .70, lo cual sugiere interrelación aceptable de los ítems. La regla Kaiser permite determinar el número de factores a seleccionar, en donde la decisión se toma con eigenvalues superiores a 1 y se complementa este criterio con la inspección visual del gráfico de sedimentación (Scree Test). Para tomar la decisión de dejar los ítems en el factor correspondiente se utilizó la carga factorial (CF) de .55 en adelante; se observó también la clara ubicación del ítem en el factor donde cargaba mayormente, y la estructura factorial que mostró que al menos cada factor integraba tres ítems en su conformación.

El análisis de regresión lineal es una técnica que permite evaluar la relación entre la variable dependiente y una o más independientes, por lo que, una vez evaluados los supuestos para su aplicación, se utilizó para validar las hipótesis de esta investigación (Ritchey, 2006). La prueba $t$-student se aplica cuando se tiene el interés de comparar las medias de dos grupos de casos y ver si existen variaciones sobresalientes (Ritchey, 2006). Para esta investigación se analizó si el personal que forma parte de las instituciones educativas estudiadas mostraba valoraciones distintas en las variables de género (hombres y mujeres) y puesto (docentes y personal administrativo).

Se aplicó la técnica de ANOVA de un factor para valorar si existen apreciaciones diferentes en la valoración de los factores determinados en la dimensión de capital humano y el desempeño por plantel educativo (CBTis 271, 236, 119 y 24). Esta técnica permite comparar medias para tres o más grupos y determina si existen diferencias significativas (Ritchey, 2006).

\section{Resultados}

\section{Características de los encuestados}

De la totalidad de los individuos que respondieron al instrumento, 55 por ciento corresponde a personal docente y el 45 por ciento restante a trabajadores de la institución (personal directivo y administrativo). Con relación al porcentaje de participación por plantel, se observó que 29 por ciento corresponde al CBTIS 271 y la misma proporción al plantel 236; 25 por ciento al CBTIS 24 y 17 por ciento al 119.

Es importante mencionar que el nivel educativo mayoritario en los planteles encuestados es licenciatura (61.2 por ciento), dato relevante dado que la intención en la institución educativa es mejorar el desempeño organizacional. Rondón (2016) menciona que el talento humano dentro de las instituciones se fortalece al desarrollar las capacidades del personal, su compromiso y su acción. Adicionalmente, la proporción entre hombres y mujeres dentro de los planteles que formaron parte del estudio piloto está proporcionada: 45 por ciento son mujeres y 55 por ciento son hombres.

El porcentaje más alto en cuanto a rango de edad ( 29 por ciento) correspondió a los encuestados de entre 34 y 41 años; así mismo, el intervalo de antigüedad más significativo se centró entre los empleados que tienen entre 6 y 10 años de trabajo en el sistema educativo. 
Estructura factorial del capital humano $y$ el desempeño educativo

Alaplicar el AFE en la variable de capital humano se identificaron tres factores con autovalores superiores a 1, conformados por ítems con cargas factoriales adecuadas (.55 en adelante), puesto que se trata de un estudio piloto y, como tal, refleja resultados preliminares. Respecto de los aspectos que agruparon los factores del constructo de capital humano, éstos se denominaron: compromiso y dinamismo (C y D), aprendizaje y colaboración (A y C), y competencia (C) (Tabla 3). Los resultados arrojados mostraron un KMO aceptable (por arriba de .70), así como la prueba de esfericidad de Bartlett ( $\mathrm{p}<.05)$, lo cual corrobora la conveniencia de la aplicación del AFE (Tabla 3). Así mismo, se confirmó un nivel de confiablidad adecuado para este constructo, con un alfa de Cronbach de .80 (Tabla 3).

Tabla 3. Factores del capital humano en CBTIS

\begin{tabular}{|c|c|c|c|c|c|}
\hline Subdimensión & Item & Media & $\mathrm{CF}$ & Alfa & AFE \\
\hline \multirow{5}{*}{$\begin{array}{l}\text { Compromiso y } \\
\text { dinamismo } \\
\text { (C y D) }\end{array}$} & $\begin{array}{l}\text { CH.9 Es capaz de desarrollar nuevas ideas y } \\
\text { conocimiento }\end{array}$ & 4.17 & .797 & \multirow[t]{11}{*}{.80} & \multirow{11}{*}{$\begin{array}{l}\text { KMO .803 } \\
\chi^{2}: 1222.944 \\
\text { gl. } 153 \\
\text { Sig. } 000\end{array}$} \\
\hline & CH.10 Es brillante y creativo & 3.85 & .654 & & \\
\hline & CH.11 Hace frente a las dificultades con eficiencia & 4.15 & .557 & & \\
\hline & $\begin{array}{l}\text { CH.12 Se centra en ofrecer un servicio de calidad } \\
\text { educativa }\end{array}$ & 4.26 & .580 & & \\
\hline & CH.16 Está comprometido con la institución & 4.40 & .551 & & \\
\hline \multirow{3}{*}{$\begin{array}{l}\text { Aprendizaje y } \\
\text { colaboración } \\
(\text { A y C) }\end{array}$} & $\begin{array}{l}\text { CH.14 Colabora con los directivos en la toma de } \\
\text { decisiones }\end{array}$ & 3.10 & .610 & & \\
\hline & $\begin{array}{l}\text { CH.15 Está satisfecho con la dirección y las políti- } \\
\text { cas de la institución }\end{array}$ & 3.73 & .794 & & \\
\hline & $\begin{array}{l}\text { CH.18 Está satisfecho con los programas y políti- } \\
\text { cas de innovación de la institución }\end{array}$ & 3.80 & .850 & & \\
\hline \multirow[t]{3}{*}{$\begin{array}{l}\text { Competencia } \\
\text { (C) }\end{array}$} & $\begin{array}{l}\text { CH.3 Cuenta con la experiencia que demanda su } \\
\text { función }\end{array}$ & 4.22 & .863 & & \\
\hline & $\begin{array}{l}\text { CH.4 Cuenta con las competencias necesarias } \\
\text { para el puesto }\end{array}$ & 4.19 & .819 & & \\
\hline & $\begin{array}{l}\text { CH.5 Cuenta con estudios profesionales acordes } \\
\text { a su función }\end{array}$ & 4.32 & .681 & & \\
\hline
\end{tabular}

Fuente: elaboración propia.

Se determinó también la conveniencia de aplicar el AFE (KMO por arriba de .70 y prueba de esfericidad de Bartlett $\mathrm{p}<.05)$ en la evaluación del constructo de desempeño (Tabla 4), y se determinó un comportamiento unidimensional, resultado que confirma lo señalado por Edvinsson y Sullivan (1996), Edvinsson y Malone (1999), y Norton y Kaplan (1993). En dicho constructo se observó que todos sus ítems mostraron carga factorial adecuada, ya que oscilaron entre .65 y .87 , con un alfa de Cronbach de .80 favorable respecto a su consistencia interna (Nunally, 1994).

Una vez valorado el instrumento se procedió a correr la técnica de regresión lineal múltiple, a fin de someter a validación la hipótesis central de esta investigación (HI): los factores del capital humano se relacionan positivamente con el desempeño de la institución educativa. 
Tabla 4. Caracterización del desempeño (D) en CBTIS

\begin{tabular}{|c|c|c|c|c|}
\hline Ítem & Media & $\mathrm{CF}$ & Alfa & AFE \\
\hline D.1 De la imagen de su institución y servicios & 3.93 & .718 & \multirow[t]{13}{*}{.93} & \multirow{13}{*}{$\begin{array}{l}\text { KMO .910 } \\
\chi^{2: 1812.148} \\
\text { gl. } 78 \\
\text { Sig. } 000\end{array}$} \\
\hline $\begin{array}{l}\text { D.2 De la calidad académica de los procesos y servicios educativos } \\
\text { proporcionados }\end{array}$ & 3.89 & .677 & & \\
\hline D.3 De la formación de calidad de los estudiantes & 3.92 & .659 & & \\
\hline D.4 Del valor añadido a los procesos-servicios otorgados & 3.87 & .734 & & \\
\hline $\begin{array}{l}\text { D.5 De la satisfacción de los usuarios con los procesos o servicios } \\
\text { proporcionados }\end{array}$ & 3.88 & .762 & & \\
\hline $\begin{array}{l}\text { D.6 Del desempeño en el logro de los objetivos e indicadores acadé- } \\
\text { micos alcanzados }\end{array}$ & 3.88 & .692 & & \\
\hline D.7 De la calidad en los procesos-servicios proporcionados & 3.78 & .758 & & \\
\hline $\begin{array}{l}\text { D.8 Del nivel de tecnología incorporado en los procesos-servicios } \\
\text { educativos }\end{array}$ & 3.70 & .677 & & \\
\hline D.9 De la productividad de nuestros empleados & 3.79 & .702 & & \\
\hline D.10 De la eficiencia en los procesos y actividades de gestión & 3.67 & .857 & & \\
\hline D.11 Del desempeño en la aplicación de los recursos & 3.58 & .853 & & \\
\hline D.12 De la mejora en la coordinación de los procesos internos & 3.58 & .878 & & \\
\hline D.13 De la innovación continua en los procesos-servicios educativos & 3.67 & .830 & & \\
\hline
\end{tabular}

Fuente: elaboración propia.

Relación entre los factores

del capital humano y el desempeño

en instituciones educativas

En primera instancia se procedió a analizar las correlaciones entre ambos constructos y se determinó que existe una asociación positiva y significativa en cada una de las tres subdimensiones del capital humano (compromiso y dinamismo (C y D), aprendizaje y colaboración (A y C) y competencia (C) con la variable dependiente de desempeño educativo (Tabla 5).

Tabla 5. Correlación entre los constructos

\begin{tabular}{lcccc}
\hline & C y D & A y C & C & D \\
\hline Correlación de Pearson & 1 & $.350^{* *}$ & $.422^{* *}$ & $.263^{* *}$ \\
\hline Sig. (bilateral) & & .000 & .000 & .000 \\
\hline
\end{tabular}

** La correlación es significativa en el nivel 0.01 (bilateral).

Fuente: elaboración propia.

Posteriormente, para validar la hipótesis central en este estudio se aplicó el análisis de regresión lineal múltiple (ARLM), y se determinó que el modelo explica en un 43.6 por ciento el desempeño educativo de las instituciones analizadas (Tabla 6). 
Tabla 6. Análisis de regresión de dimensiones: capital humano y desempeño

\begin{tabular}{|c|c|c|c|c|c|c|c|c|c|c|}
\hline \multirow[t]{2}{*}{ Modelo } & \multirow[t]{2}{*}{$\mathrm{R}$} & \multirow[t]{2}{*}{$\mathrm{R}^{2}$} & \multirow[t]{2}{*}{$\mathrm{R}^{2}$ ajustado } & \multirow{2}{*}{$\begin{array}{c}\text { Error es- } \\
\text { tándar de la } \\
\text { estimación }\end{array}$} & \multicolumn{5}{|c|}{ Estadísticos de cambio } & \multirow{2}{*}{$\begin{array}{l}\text { Durbin- } \\
\text { Watson }\end{array}$} \\
\hline & & & & & $\begin{array}{l}\text { Cam- } \\
\text { bio en } \\
\mathrm{R}^{2}\end{array}$ & $\begin{array}{l}\text { Cam- } \\
\text { bio } \\
\text { en F }\end{array}$ & gl 1 & $\mathrm{gl}^{2}$ & $\begin{array}{c}\text { Sig. } \\
\text { cambio } \\
\text { en F }\end{array}$ & \\
\hline 1 & .663 & .439 & .436 & .73310388 & .439 & 137.881 & 1 & 176 & .000 & 2.129 \\
\hline
\end{tabular}

a. Predictores: (constante), A y C.

b. Variable dependiente: D.

Fuente: elaboración propia.

Los resultados permiten observar que la dimensión de aprendizaje y colaboración (A y C) mostró en mayor medida la contribución a la variable dependiente de desempeño (D) educativo (.772, Pv=.000). En contraste, los factores de compromiso y dinamismo (C y D), así como el de competencia (C) no aportan a explicar la variable desempeño (D). De esta forma se confirma parcialmente la hipótesis, puesto que no todos los factores contribuyen; la implicación para la gerencia educativa es trazar estrategias que impulsen al activo intangible en áreas de aprendizaje y colaboración.

\section{Contrastes en la valoración}

de las dimensiones del capital humano $y$ el desempeño educativo

Con el interés de cumplir con los objetivos específicos de esta indagación, referente a si existen valoraciones distintas de los constructos estudiados respecto al género y tipo de puesto de los encuestados, se procedió a correr la prueba $t$. De esta forma se corroboró que no existen diferencias entre hombres y mujeres, ni entre administrativos y docentes, puesto que todos califican con el mismo nivel de importancia las dimensiones del capital humano y del desempeño en el presente estudio (Tabla 7).

Tabla 7. Valoración del capital humano y desempeño desde la percepción de los encuestados

\begin{tabular}{|c|c|c|c|c|c|}
\hline \multirow[b]{2}{*}{ Sexo } & \multirow[b]{2}{*}{ Estadísticos } & \multicolumn{3}{|c|}{ Capital humano } & \multirow{2}{*}{$\begin{array}{c}\text { Desempeño } \\
\text { D }\end{array}$} \\
\hline & & C y D & $\mathrm{A}$ y C & C & \\
\hline \multirow[t]{2}{*}{ Mujeres } & Media & 4.183 & 3.503 & 4.177 & 3.825 \\
\hline & Desviación típica & .6154 & 1.0362 & .7932 & .5863 \\
\hline \multirow[t]{4}{*}{ Hombres } & Media & 4.147 & 3.621 & 4.346 & 3.752 \\
\hline & Desviación típica & .5619 & .8950 & .5869 & .7138 \\
\hline & $t$ & -.400 & .795 & 1.577 & -.749 \\
\hline & $\mathrm{Pv}$ & .690 & .427 & .117 & .455 \\
\hline \multirow[t]{2}{*}{ Docentes } & Media & 4.145 & 3.576 & 4.296 & 3.742 \\
\hline & Desviación típica & .5472 & .9675 & .6545 & .5828 \\
\hline \multirow[t]{4}{*}{ Administrativos } & Media & 4.190 & 3.506 & 4.181 & 3.826 \\
\hline & Desviación típica & .6492 & .9969 & .7859 & .5828 \\
\hline & $t$ & -.495 & .469 & 1.064 & -.847 \\
\hline & $\mathrm{Pv}$ & .621 & .639 & .289 & .398 \\
\hline
\end{tabular}

Fuente: elaboración propia. 
En este sentido, sobre la base de los hallazgos se determinó que tanto los hombres como las mujeres participantes valoran con el mismo nivel de importancia que la mayoría de las veces son capaces de desarrollar nuevas ideas y de aplicar sus conocimientos en sus instituciones educativas; que saben hacer frente a las dificultades con eficiencia; que se enfocan a proporcionar servicios de calidad y están comprometidos con su escuela. También manifiestan que la mayoría de las veces colaboran con los directivos en la toma de decisiones, además de considerar que el personal cuenta, en la mayoría de los casos, con las competencias (experiencia, escolaridad) que demanda el puesto. Estos aspectos que valoran hombres y mujeres activos en el servicio educativo de las instituciones analizadas son de suma importancia para que la gerencia educativa los tome en cuenta en el diseño e implementación de estrategias de retención y desarrollo profesional de su capital humano, sobre todo porque tanto hombres como mujeres estiman que los empleados son recursos intangibles que contribuyen a un buen desempeño educativo en lo relativo a la imagen institucional, la calidad académica de los servicios y procesos educativos, y la satisfacción de los usuarios, así como respecto de la obtención de indicadores y resultados educativos en los planteles educativos analizados.

En lo que respecta a los resultados de contraste en la variable puesto (docentes y administrativos), en los encuestados de los cuatro planteles educativos, ambas categorías laborales valoran con el mismo nivel de importancia los aspectos relacionados con los factores determinantes del capital humano, que fueron compromiso y dinamismo, aprendizaje y colaboración, y competencia del personal; así mismo, docentes y administrativos valoran con la misma importancia todos los indicadores que se estiman relevantes para el desempeño organizacional de escuelas tecnológicas de educación media superior, como son su imagen institucional, la calidad y eficiencia de los servicios y procesos que llevan a cabo, la satisfacción de sus usuarios, la consecución de sus objetivos y metas académicas, y la formación de calidad de sus estudiantes, por mencionar sólo algunos de los indicadores que caracterizaron este constructo de desempeño en los CBTIS (Tabla 4).

Adicionalmente, al evaluar las dimensiones de capital humano y desempeño por tipo de plantel educativo estudiado, los resultados del ANOVA mostraron que solamente en dos factores se identifican diferencias sobresalientes en el nivel de importancia que dan los encuestados:

Tabla 8. ANOVA percepción del capital humano por plantel

\begin{tabular}{|c|c|c|c|c|c|}
\hline \multirow[b]{2}{*}{ Plantel } & \multirow[b]{2}{*}{ Estadísticos } & \multicolumn{3}{|c|}{ Capital humano } & \multirow{2}{*}{$\begin{array}{c}\text { Desempeño } \\
\text { D }\end{array}$} \\
\hline & & C y D & A y C & $\mathrm{C}$ & \\
\hline \multirow[t]{2}{*}{271} & Media & 4.086 & 3.085 & 4.170 & 3.480 \\
\hline & Desviación típica & .6815 & .9952 & .6712 & .5936 \\
\hline \multirow[t]{2}{*}{236} & Media & 4.161 & 3.490 & 4.137 & 3.830 \\
\hline & Desviación típica & .5987 & .9436 & .9526 & .6206 \\
\hline \multirow[t]{2}{*}{119} & Media & 4.047 & 3.889 & 4.222 & 3.774 \\
\hline & Desviación típica & .4776 & .7895 & .5129 & .4620 \\
\hline \multirow[t]{4}{*}{24} & Media & 4.335 & 3.891 & 4.464 & 4.059 \\
\hline & Desviación típica & .5267 & .9110 & .5187 & .7366 \\
\hline & Valor F & 1.986 & 7.765 & 2.049 & 7.130 \\
\hline & Sig & .118 & .000 & .109 & .000 \\
\hline
\end{tabular}

Fuente: elaboración propia. 
aprendizaje y colaboración, y desempeño. Ello permite afirmar que los encuestados adscritos al plantel 24 valoran más alto el aprendizaje y colaboración que los de la escuela 271, y en la variable de desempeño es el mismo comportamiento. En las otras dos dimensiones no se observan diferencias significativas (Tabla 8).

\section{CONCLUSIONES Y FUTURAS INVESTIGACIONES}

Actualmente se vive una época en la que las instituciones educativas del sector público deben interesarse en generar mediciones adecuadas de su desempeño a fin de que los recursos y beneficios asignados por parte del Estado se justifiquen. El objetivo central del presente estudio fue conocer si existe una relación significativa entre los factores del capital humano y el desempeño educativo.

En la presente investigación pudo confirmarse que el capital humano tiende a contribuir positivamente hacia el desempeño de los planteles educativos, hallazgo que coincide con estudios previos (Pedraza, 2020); sin embargo, se observó que no todas sus dimensiones aportan a esta variable dependiente, ya que sólo el factor de aprendizaje y colaboración influye significativamente en el desempeño educativo. Este hallazgo concuerda con lo afirmado por autores como Horn y Murillo (2016), quienes determinaron que acciones claras e incluyentes por parte de los directivos escolares favorecen el desarrollo del capital humano educativo y que éste, a su vez, influye en el desempeño institucional.

Adicionalmente, y en respuesta a los objetivos específicos, se determinó, desde la percepción de los encuestados, que ni el género ni el puesto tienen diferencias importantes en la valoración de los factores del capital humano y el desempeño educativo. Caso contrario, al aplicar el ANOVA para analizar las diferencias ente los cuatro planteles se observó que el personal del CBTIS 24 valora más alto el aprendizaje y la colaboración, así como el desempeño, en comparación con el resto de los planteles analizados.

Los resultados muestran que el personal de las instituciones se encuentra mayormente interesado en la colaboración con los directivos y con las políticas y programas de innovación, mismas que coadyuvan en la mejora del desempeño de las instituciones. Esto coincide con los resultados obtenidos por Ayaz et al. (2018), quienes determinaron que una adecuada gestión en la colaboración de los miembros escolares permite incrementar el rendimiento y la efectividad escolar; así como con los de Villagra et al. (2018), quienes encontraron un impacto significativo en el desempeño educativo cuando se cuenta con las condiciones laborales adecuadas; finalmente, Siu (2020) es contundente al determinar que estos aspectos favorecen hasta en un 70 por ciento la mejora en el desempeño educativo de las instituciones. Estos hallazgos permiten que la gerencia educativa identifique áreas de oportunidad para trazar políticas y estrategias de gestión del talento humano en el campo del aprendizaje y la colaboración.

El presente estudio se aplicó en cuatro planteles pertenecientes a la UEMSTIS en Ciudad Victoria, Tamaulipas (México), por ende, los resultados tienen la principal implicación de que su implementación corresponde a estas cuatro unidades de análisis; dado lo anterior, se sugiere que estudios futuros sean aplicados a muestras más grandes del mismo contexto a fin de poder observar tendencias en el análisis del objeto de estudio. Lo anterior se refuerza porque en esta investigación, 40 por ciento de los encuestados señaló que es importante colaborar con los directivos en la toma de decisiones; 65 por ciento manifestó estar satisfecho con la dirección y las políticas de su institución; y 67 por ciento menciona estar de acuerdo con los programas y políticas de innovación.

La principal contribución de este estudio es marcar una pauta para el análisis de los activos intangibles en la gestión y desempeño de instituciones educativas, a fin de contribuir a 
generar conocimiento que sea útil a la mejora del desempeño académico en instituciones de educación media superior, que son entes con la gran responsabilidad de formar personas altamente capacitadas que se habrán de insertar tarde o temprano en el mercado laboral.

\section{REFERENCIAS}

Agasisti, Tommaso, Cristian Barra y Roberto Zotti (2016), "Evaluating the Efficiency of Italian Public Universities (2008-2011) in Presence of (Unobserved) Heterogeneity", Socio-Economic Planning Sciences, vol. 55, pp. 47-58. DOI: https://doi.org/10.1016/j.seps.2016.06.002

Allui, Alwiya y Jolly Sahni (2016), "Strategic Human Resource Management in Higher Education Institutions: Empirical evidence from Saudi", Procedia - Social and Behavioral Sciences, vol. 235, núm. 2, pp. 361-371. DOI: https://doi. org/10.1016/j.sbspro.2016.11.044

Arechavala Vargas, Ricardo y Claudia Fabiola Sánchez Cervantes (2017), "Las universidades públicas mexicanas: los retos de las transformaciones institucionales hacia la investigación y la transferencia de conocimiento", Revista de la Educación Superior, vol. 46, núm. 184, pp. 21-37.

Awan, Abdul Ghafoor y Saeed Kashif (2014), "Intellectual Capital and Research Performance of Universities in Southern Punjab-Pakistan", European Journal of Business and Innovation Research, vol. 2, núm. 6, pp. 21-39.

Ayaz, Mohammad Qasim, Qahar Abdul y Ullah Raza (2018), “Assessing the Influence of Selected Human Capital Management Factors on the Organizational Performance of Higher Education Sector in Afghanistan", Journal of Business and Tourism, vol. 4, núm. 2, pp. 173-180.

Bahrami, Susan, Rajaeepour Saeed, Hossein Yarmohmmadian Mohammad y Ali Bakhtiar Narsabadi Hassan (2013), "Simple and Multiple Relations between Strategic Human Resource Management and Intellectual Capital in Iranian Higher Education", African Journal of Business Management, vol. 7, núm. 35, pp. 3451-3460. DOI: https://doi.org/10.5897/AJB M12.104

BARbosA-Chacón, Jorge Winston, Juan Carlos Barbosa Herrera y Margarita Rodríguez Villabona (2015), "Concepto, enfoque y justificación de la sistematización de experiencias educativas. Una mirada 'desde' y 'para' el contexto de la formación universitaria”, Perfiles Educativos, vol. 37, núm. 149, pp. 130-149.

Barreto, Ariel y Armando Azeglio (2013), "La problemática de la gestión del capital humano en las MiPyMEs de alojamiento turístico de la ciudad de Buenos Aires-Argentina", Estudios y Perspectivas en Turismo, vol. 22, núm. 6, pp. 1140-1159, en: http://www.scielo.org.ar/ scielo.php?pid $=\$ 185117322013000600007 \&$ scri pt=sci_arttext (consulta: marzo de 2019).

Bontis, Nick (1998), "Intellectual Capital: An exploratory study that develops measures and models", Management Decision, vol. 36, núm. 2, pp. 63-76.

Bontis, Nick (2001), "Assessing Knowledge Assets: A review of the models used to measure intellectual capital", International Journal of $\mathrm{Ma}$ nagement Reviews, vol. 3, núm. 1, pp. 41-60.

Bontis, Nick y Alejandro Serenko (2009), "A Causal Model of Human Capital Antecedents and Consequents in the Financial Services Industry", Journal of Intellectual Capital, vol. 10, núm. 1, pp. 53-69. DOI: https://doi. org/10.1108/14691930910922897

Broucker, Bruno, Kurt De Wit y Jef C. Verhoeven (2017), "Higher Education Research: Looking beyond new public management', Theory and Method in Higher Education Research, vol. 3, pp. 21-38.

Bueno, Eduardo, Ma. Paz Salmador y Carlos Merino (2008), "Génesis, concepto y desarrollo del capital intelectual en la economía del conocimiento: una reflexión sobre el modelo Intellectus y sus aplicaciones”, Estudios de Economía Aplicada, vol. 26, núm. 2, pp. 43-63.

CABrol, Marcelo y Miguel Székely (eds.) (2012), Educación para la transformación, Washington D.C., BID.

Castro Valles, Alberto (2015), "Competencias administrativas y académicas en el profesorado de educación media superior", Revista Mexicana de Investigación Educativa, vol. 22, núm. 64, pp. 263-294.

Centzal Peniche, Rubí Surema, Cristóbal Ramón Mac, Cintya Guzmán Ramírez y Noé Mora Ozuna (2020), "Factores que afectan el desempeño docente en centros de alta y baja eficacia en México", REICE: Revista Iberoamericana sobre Calidad, Eficacia y Cambio en Educación, vol. 18, núm. 2, pp. 77-95. DOI: https:// doi.org/10.15366/reice2020.18.2.004

Chatterji, Niti y Ravi Kiran (2017), "Role of Human and Relational Capital of Universities as Underpinnings of a Knowledge Economy: A structural modelling perspective from north Indian universities", International Journal of Educational Development, vol. 56, pp. 52-61. DOI: https://doi.org/10.1016/j.ijedu dev.2017.06.004 
Chawla, Saniya y Usha Lenka (2015), "A Study on Learning Organizations in Indian Higher Educational Institutes", Journal of Workplace Learning, vol. 27, núm. 2, pp. 142-161. DOI: https://doi.org/10.1108/JWL-07-2014-0052

Cheng, Eric C.K. (2015), "Knowledge Sharing for Creating School Intellectual Capital”, Procedia - Social and Behavioral Sciences, vol. 191, núm. 852, pp. 1455-1459. DOI: https://doi.org /10.1016/j.sbspro.2015.04.307

Cóndor Quimbita, Beatriz Hortencia y Manuel Remache Bunci (2019), "La evaluación al desempeño directivo y docente como una oportunidad para mejorar la calidad educativa", Cátedra, vol. 2, núm. 1, pp. 116-131.

Creswell, John W., Ann Carroll Klassen, Vicki Plano y Katherine Clegg Smith (2013), "Best Practices for Mixed Methods Research in the Health Sciences", National Institutes of Health, vol. 12, núm. 4, pp. 541-545.

Cuentas Martel, Susan Carol (2018), “La gestión del capital humano en una unidad académica de educación superior", Educación, vol. 27, núm. 53, pp. 57-72. DOI: https://doi.org/10. 18800/educacion.201802.004

Dal Molin, Martina, Mateo Turri y Tommaso Agasisti (2017), "New Public Management Reforms in the Italian Universities: Managerial tools, accountability mechanisms or simply compliance?", International Journal of Public Administration, vol. 40, núm. 3, pp. 256-269.

Decker, Paul T., Daniel P. Mayer y Steven Glazerman (2004), "The Effects of Teach for America on Students: Findings from a national evaluation", Wisconsin, University of Wisconsin-Madison-Institute for Research on Poverty.

Edvinsson, Leif y Michael Malone (1999), El capital intelectual. Cómo identificar y calcular el valor de los recursos intangibles de su empresa, Barcelona, Gestión 2000.

Edvinsson, Leif y Patrick Sullivan (1996), "Developing a Model for Managing Intellectual Capital”, European Management Journal, vol. 14, núm. 4, pp. 356-364.

Farah, Assaad y Shadi Abouzeid (2017), "The Impact of Intellectual Capital on Performance: Evidence from the public sector", Knowledge Management \& E-Learning, vol. 9, núm. 2, pp. 225-238.

FAZlagic, Amir (2005), "Measuring the Intellectual Capital of a University", In Trends in the Management of Human Resources in Higher Education, París, OCDE, en: www.oecd.org /education/imhe/35322785.pdf (consulta: marzo de 2019).

Flores Paz, Teresa Mariela (2020), "Gestión educativa y la mejora del desempeño laboral de los docentes", Ciencia y Educación-Revista Científica, vol. 1, núm. 4, pp. 15-26.
Fullan, Michael (2019), "Liderar los aprendizajes: acciones concretas en pos de la mejora escolar", Revista Eletrônica de Educação, vol. 13, núm. 1, pp. 58-65. DOI: https://doi.org/10. 14244/198271993074

Gómez,Luis F.y María G. Valdés (2019), “La evaluación del desempeño docente en la educación superior", Propósitos y Representaciones, vol. 7, núm. 2, pp. 479-515. DOI: https://doi.org/10. 20511/pyr2019.v7n2.255

González Fernández, María Obdulia (2018), “Percepción del desempeño docente-estudiante en la modalidad mixta desde una mirada ecosistémica", RIDE, Revista Iberoamericana para la Investigación y el Desarrollo Educativo, vol. 8, núm. 16, pp. 370-393. DOI: https:// doi.org/10.23913/ride.v8i16.346

GonzÁLEz-Loureiro, Miguel y Antonio Moreira Teixeira (2011), "Intellectual Capital in Public Universities: A performance-oriented approach to manage intangible", International Journal of Engineering and Industrial $\mathrm{Ma}$ nagement, vol. 3, núm. 1, pp. 95-125.

Guthrie, James y John Dumay (2015), "New Frontiers in the Use of Intellectual Capital in the Public Sector", Journal of Intellectual Capital, vol. 16, núm. 2, pp. 258-266. DOI: https:// doi.org/10.1108/JIC-02-2015-0017

Guzmán Fernández, Candelaria, Norma Aguilar Morales, Deneb Elí Magaña Medina y Germán Martínez Prats (2020), “Autonomía de la gestión escolar en la educación básica en México: identificación de factores que la obstaculizan o permiten su desarrollo", Revista Inclusiones, vol. 7, núm. especial, pp. 62-83.

GuZmán, Isabel y Rigoberto Marín (2011), "La competencia y las competencias docentes: reflexiones sobre el concepto y la evaluación", Revista Electrónica Interuniversitaria de Formación del Profesorado, vol. 14, núm. 1, pp. 151-163, en: https://www.redalyc.org/pdf /2170/217017192012.pdf (consulta: marzo de 2019).

HaIR, Joseph, William Black, Barry J. Babin, Rolph E. Anderson (2014), Multivariate Data Analysis, Edimburgo, Pearson Education Limited.

Hernández-Sampieri, Roberto y Christian Paulina Mendoza Torres (2018), Metodología de la investigación, vol. 4, México, McGraw-Hill Interamericana.

Hili, Padli, Mursalim Umar Gani, Nazir Hamzah y Zainuddin Rahman (2017), "Effect of $\mathrm{Hu}$ man Capital and Leadership on Institutions' Performance and Competitive Advantages", IRA-International Journal of Management \& Social Sciences, vol. 7, núm. 3, pp. 479-488. DOI: https://doi.org/10.21013/jmss.v7.n3.p9 
Hill, Peter W. y Kenneth J. Rowe (1996), "Multilevel Modeling in School Effectiveness Research", International Journal Research, Policy and Practice, vol. 12, núm. 1, pp. 1-34. DOI: https://doi.org/10.1080/0924345960070101

Horn Kupfer, Andrea y Francisco Murillo Torrecilla (2016), "Incidencia de la dirección escolar sobre el compromiso de los docentes: un estudio multinivel", Psicoperspectivas, vol. 15, núm. 2, pp. 64-77, en: https://www.psicoperspectivas.cl/index.php/psicoperspectivas/article/view/746 (consulta: marzo de 2019).

Khalkhali, Ali, Zohreh Shakibaei y R. Khodadoost (2012), "Designing a Model to Recognize and Manage Intellectual Capital in Education System", Procedia-Social and Behavioral Sciences, vol. 46, pp. 992-997.

Kelly, Anthony (2004), "The Intellectual Capital of Schools: Analyzing government policy statements on school improvement in light of a new theorization", Journal of Education Policy, vol. 19, núm. 5, pp. 609-629. DOI: https:// doi.org/10.1080/0268093042000269180

Kucharčíková, Alzbeta, Emese Tokarčíková y Martina Blašková (2015), "Human Capital Management -Aspect of the Human Capital Efficiency in University Education", Procedia - Social and Behavioral Sciences, vol. 177, pp. 48-60. DOI: https://doi.org/10.1016/j.sbs pro.2015.02.332

LeIšYté, Liudvika (2016), “New Public Management and Research Productivity -A Precarious State of Affairs of Academic Work in the Netherlands", Studies in Higher Education, vol. 41, núm. 5, pp. 828-846. DOI: https://doi. org/10.1080/03075079.2016.1147721

Leitner, Karl-Heinz (2004), "Intellectual Capital Reporting for Universities Conceptual Background and Application for Austrian Universities", Research Evaluation, vol. 13, núm. 2, pp. 129-140.

Leiva-Guerrero, María Verónica y Camila Vásquez (2019), "Liderazgo pedagógico: de la supervisión al acompañamiento docente", Calidad en la Educación, vol. 51, pp. 225-251.

López Leyva, Santos (2016), "Competitividad de la educación superior en cuatro países de América Latina: perspectiva desde un ranking mundial", Revista de la Educación Superior, vol. 45, núm. 178, pp. 45-59. DOI: https://doi. org/10.1016/j.resu.2016.02.003

Lora Elizalde, Leticia, Patricia Bezies Cruz y Brenda Ivonne Olvera Larios (2018), "Detección de variables predictoras de éxito y desempeño desfavorable de la actividad docente en pregrado presencial en una institución pública estatal", Edetania, vol. 54, pp. 73-92.
Mallama Casas, Angela (2019), "Liderazgo pedagógico, nuevas perspectivas para el desempeño docente", Investigación Valdizana, vol. 13, núm. 1, pp. 51-60. DOI: https://doi.org/10.33554 /riv.13.1.171

Martín Linares, Xiomara, Alina María Segredo Pérez e Irene Perdomo Victoria (2013), "Capital humano, gestión académica y desarrollo organizacional", Human Capital, Academic Management and Organizational Development, vol. 27, núm. 3, pp. 288-295.

Martínez-Chairez, Guadalupe Iván y Albertico Guevara-Araiza (2015), "La evaluación del desempeño docente”, Ra Ximhai, vol. 11, núm. 4, pp. 113-124, en: https://www.redalyc. org/pdf/461/46142596007.pdf (consulta: febrero de 2019).

Morales Clark, Laura Elena, Carlos Armando Jacobo-Hernández, Sergio Ochoa-Jiménez y Luis Enrique Ibarra-Morales (2020), "Capital intelectual y desempeño organizacional: el caso de las instituciones de educación básica en México", Revista Científica Pensamiento y Gestión, vol. 47, núm. 47, en: http:// www.scielo.org.co/scielo.php?pid=\$1657-6276 2019000200180\&script=sci_abstract\&tlng=es (consulta: marzo de 2019).

Morales, Rodrigo, Ricardo Morales y Morayma Jimena Bustos Yépez (2017), "Competencias docentes y desempeño: un estudio con profesores universitarios", Revista Publicando, vol. 4, núm. 1, pp. 248-269.

Morgado, Carmen, Julio Juvenal Aldana Zavala y Josía Isea Argüelles (2019), "Gerencia transformacional desde el accionar docente directivo para el abordaje del contexto escolar", Cienciamatria, vol. 5, núm. 9, pp. 48-72.

MüNCH Galindo, Lourdes y Ernesto Ángeles (2009), Métodos y técnicas de investigación, México, Trillas.

Nava-Rogel, Rosa María y Patricia Mercado-Salgado (2011), "An Analysis of the Trajectory of Intellectual Capital in a Mexican Public University", Revista Electrónica de Investigación Educativa, vol. 13, núm. 2, pp. 166-187, en: http://www.scopus.com/inward/record. url?eid=2-s2.0 $-82955168467 \&$ partnerID $=40$ \&md5=f7db1febcf9972bdc2f7e5599c27609d (consulta: febrero de 2019).

Norton, David y Robert Kaplan (1993), "Evaluación de los resultados: algo más que números”, Harvard Deusto Business Review, vol. 55, núm. 3, pp. 18-25.

Nunnally, Jum C. (1995), Teoría psicométrica, México, McGraw Hill.

OCDE (2017), Estudios económicos de la OCDE, París, OCDE.

OCDE (2018), “OECD Labour Force Statistics 2017”, París, OCDE. 
OrdoñEz Espinoza, Cristina Guadalupe, Deysi Carolina Castillo Castillo, Ana Luisa del Rocío Ordoñez Laso y Mariory Prisila Orbe Guaraca (2020), "Liderazgo directivo y desempeño docente: abordaje desde el ámbito legal ecuatoriano", Journal of Business and Entrepreneurial Studies, vol. 4, núm. 1, en: http://portal. amelica.org/ameli/jatsRepo/383/3831589012/ index.html (consulta: marzo de 2019).

Orozco Encinas, Francisca Cecilia (2014), “Orientación de las organizaciones públicas al aprendizaje organizacional. El caso de los organismos descentralizados en el estado de Sonora, México", Estudios Gerenciales, vol. 30, núm. 130, pp. 10-17. DOI: https://doi.org/10.1016/j.estger. 2014.01.010

Pedraza Melo, Norma, Jesús Lavín Verástegui, Jesús Marmolejo Rodríguez, Román Vasconcelos Álvarez y Jesús García Ruiz (2017), "Caracterización del capital intelectual en el sector empresarial del estado de Tamaulipas, México", Visión Gerencial, vol. 1, pp. 119-143

PedrazA, Norma (2020), "Satisfacción laboral y compromiso organizacional del capital humano en el desempeño de instituciones de educación superior", RIDE. Revista Iberoamericana para la Investigación y Desarrollo Educativo, vol. 10, núm. 20, e051. DOI: https://doi. org/10.23913/ride.v10i20.595

PÉrez, Edgardo y Leonardo Adrián Medrano (2010), "Análisis factorial exploratorio: bases conceptuales y metodológicas", Revista Argentina de Ciencias del Comportamiento, vol. 2, núm. 1, pp. 58-66.

Pérez, Olga y Jorge Pino (2018), "Rol de la gestión educativa estratégica en la gestión del conocimiento, la ciencia, la tecnología y la innovación en la educación superior", Educación Médica, vol. 19, núm. 1, pp. 51-55. DOI: https://doi.org/10.1016/j.edumed.2016.12.001

Perez Lopez, Susana, Jose Manuel Montes Peon y Camilo Jose Vazquez Ordas (2005), "Human Resource Practices, Organizational Learning and Business Performance", Human Resource Development International, vol. 8, núm. 2, pp. 147-164.

PÉrez Segredo, Alina María (2016), "Desarrollo organizacional. Una mirada desde el ámbito académico", Educación Médica, vol. 17, núm. 1, pp. 3-8.

Queiroz, Alexandre Bossi, Yolanda Fuertes Callen y Carlos Serrano Cinca (2005), "Reflexiones en torno a la aplicación del capital intelectual en el sector público", Revista Española de Financiación y Contabilidad, vol. 34, núm. 124, pp. 211-245.

Quispe Pareja, Marleni (2020), "La gestión pedagógica en la mejora del desempeño docente", Investigación Valdizana, vol. 14, núm. 1, pp. 7-14. DOI: https://doi.org/10.33554/riv.14.1.601
Ramirez, Yolanda y Silvia Gordillo (2014), “Recognition and Measurement of Intellectual Capital in Spanish Universities", Journal of Intellectual Capital, vol. 15, núm. 1, pp. 173-188. DOI: https://doi.org/10.1108/JIC-05-2013-0058

Ramirez, Yolanda, Carmen Lorduy y José Antonio Rojas (2007), "Intellectual Capital Management in Spanish Universities", Journal of IntellectualCapital,vol. 8, núm.4,pp.732-748. DOI: https://doi.org/10.1108/14691930710830873

Ritchey, Ferris J. (2006), Estadística para las ciencias sociales, México, McGraw-Hill.

Rojas, Mariano, Humberto Angulo e Irene Velázquez (2000), "Rentabilidad de la inversión en capital humano en México”, Economía Mexicana Nueva Época, vol. 9, núm. 2, pp. 113-142.

Rondón, Musa Ammar Majad (2016), "Gestión del talento humano en organizaciones educativas", Revista de Investigación, vol. 40, núm. 88, pp. 148-165.

Rowe, Kenneth, Peter Hill y Philip Holmes-Smith (1995), "Methodological Issues in Educational Performance and School Effectiveness Research: A discussion with worked examples", Australian Journal of Education, vol. 39, núm. 3, pp. 217-248.

Sánchez, M. Paloma, Susana Elena y Rocio Castrillo (2009), "Intellectual Capital Dynamics in Universities: A reporting model”, Journal of Intellectual Capital, vol. 10, núm. 2, pp. 307-324. DOI: https://doi.org/10.1108/14691930910952687

Segatto, Catarina Ianni y Fernando Luiz Abrucio (2017), “A gestão por resultados na educação em quatro estados brasileiros", Revista do Serviço Público, vol. 68, núm.1, pp. 85-106.

Siu Rodríguez, Jenny Liliana (2020), "Las habilidades blandas como base del buen desempeño del docente universitario", Innova Research Journal, vol. 5, núm. 2, pp. 186-199. DOI: https:// doi.org/10.33890/innova.v5.n2.2020.1321

Sveiby, Karl Erik (1997), The New Organizational Wealth, Nueva York, Berret-Koehler.

ToAlá-Sánchez, Glenda, Doris Meza-Bolaños y Gina Mejía-Madrid (2017), "Modelo de gestión del conocimiento y capital intelectual de los docentes universitarios", Revista Publicando, vol. 4, núm. 11, pp. 270-286.

VIDAL Tovar, Carlos Ramón (2017), "Modelo de capital intelectual para la investigación en las universidades públicas de la Costa Caribe colombiana", Actualidades Investigativas en Educación, vol. 17, núm. 1, pp. 400-427.

Villagra, Carolina, María Elena Mellado Hernández y Sixto Cubo Delgado (2018), "Relación entre variables mediadoras del desempeño docente y resultados educativos: una aproximación al liderazgo escolar”, Opción, vol. 34, núm. 87, pp. 213-240. 
Webster, Jane y Richard T. Watson (2002), "Analyzing the Past to Prepare for the Future: Writing a literature review", Management Information Systems Research Center, vol. 26, núm. 2, pp. 13-23.

Wu, Hung-Yi, Jui-Kuei Chen, I-Shuo Chen y HsinHui Zhuo (2012), "Ranking Universities Based on Performance Evaluation by a Hybrid MCDM Model", Measurement, vol. 45, núm. 5, pp. 856-880. DOI: https://doi.org/10.1016/j. measurement.2012.02.009

Xu, Zeyu, Jane Hannaway y Colin Taylor (2011), "Making a Difference? The Effects of Teach for America in High School", Journal of Policy Analysis and Management, vol. 30, núm. 3, pp. 447-469. DOI: https://doi.org/10.1002/ pam.20585

Zlate, Ştefania y Cerasela Enache (2015), "The Interdependence Between Human Capital and Organizational Performance in Higher Education", Procedia -Social and Behavioral Sciences, vol. 180, pp. 136-143. DOI: https:// doi.org/10.1016/j.sbspro.2015.02.096 
AneXo: InSTRUMENTO DE

\section{RECOLECCIÓN DE DATOS}

Objetivo. El presente cuestionario se aplica con el propósito de identificar en su institución, la gestión del capital humano y su relación con la competitividad educativa en el estado de Tamaulipas, específicamente en los Centros de Bachilleratos Tecnológicos, Industrial y de Servicios (CBTIS). La información proporcionada será para fines académicos, y se garantiza total confidencialidad de la misma.

Considere que el capital humano $(\mathrm{CH})$ se refiere a las competencias del personal, a la capacidad de aprender, a las habilidades y experiencia con la que cuentan para generar valor a los procesos y servicios de su institución. Por otro lado, la competitividad educativa (CE) se puede definir como aquellos efectos resultantes de la gestión de estos activos intangibles que permiten mejorar los resultados e indicadores educativos logrados.

SECCION UNO. Instrucciones. Marcar con una " $X$ " su respuesta sobre el número que más refleja la manera como usted percibe la situación expuesta en su institución en los últimos tres años. Si no está seguro, por favor, elija aquella opción que más se aproxime. Se agradece responder todas las preguntas. $\mathrm{Su}$ opinión es valiosa.

\begin{tabular}{lll}
\hline $1=$ Nunca & $2=$ Rara vez & $3=$ Algunas veces \\
\hline $4=$ La mayoría de las veces & $5=$ Siempre \\
\hline
\end{tabular}

\begin{tabular}{|c|c|c|c|c|c|}
\hline \multicolumn{6}{|l|}{ Capital humano (el personal de esta institución...) } \\
\hline $\mathrm{CH} 1 . .$. aprende continuamente de otros & 1 & 2 & 3 & 4 & 5 \\
\hline $\begin{array}{l}\mathrm{CH} 2 . . . \text { es capaz de participar en la flexibilidad laboral (rotación del puesto, enriquecimiento } \\
\text { del puesto, rotación de cátedra) }\end{array}$ & 1 & 2 & 3 & 4 & 5 \\
\hline $\mathrm{CH} 3 . .$. cuenta con la experiencia que demanda su función & 1 & 2 & 3 & 4 & 5 \\
\hline CH4.... cuenta con las competencias necesarias para el puesto & 1 & 2 & 3 & 4 & 5 \\
\hline CH5.... cuenta con estudios profesionales acordes a su función & 1 & 2 & 3 & 4 & 5 \\
\hline $\begin{array}{l}\text { CH6.... colabora entre sí para resolver problemas y desarrollar soluciones a los procesos y } \\
\text { servicios educativos }\end{array}$ & 1 & 2 & 3 & 4 & 5 \\
\hline CH7. ... comparte recursos e información de los procesos y servicios educativos & 1 & 2 & 3 & 4 & 5 \\
\hline CH8. ... obtiene mejores resultados cuando trabajan en equipo & 1 & 2 & 3 & 4 & 5 \\
\hline CH9. ... es capaz de desarrollar nuevas ideas y conocimiento & 1 & 2 & 3 & 4 & 5 \\
\hline $\mathrm{CH} 10 . .$. es brillante y creativo & 1 & 2 & 3 & 4 & 5 \\
\hline CH11. ... hace frente a las dificultades con eficiencia & 1 & 2 & 3 & 4 & 5 \\
\hline $\mathrm{CH} 12 . .$. se centra en ofrecer un servicio de calidad educativa & 1 & 2 & 3 & 4 & 5 \\
\hline $\mathrm{CH} 13 . .$. tiene un bajo índice de rotación & 1 & 2 & 3 & 4 & 5 \\
\hline $\mathrm{CH} 14 . .$. colabora con los directivos en la toma de decisiones & 1 & 2 & 3 & 4 & 5 \\
\hline CH15.... está satisfecho con la dirección y las políticas de la institución & 1 & 2 & 3 & 4 & 5 \\
\hline CH16. ... está comprometido con la institución & 1 & 2 & 3 & 4 & 5 \\
\hline $\mathrm{CH} 17 . .$. tiene la confianza de colaborar en equipos de trabajo & 1 & 2 & 3 & 4 & 5 \\
\hline CH18. ... está satisfecho con los programas y políticas de innovación de la institución & 1 & 2 & 3 & 4 & 5 \\
\hline
\end{tabular}


SECCION DOS. Instrucciones. Marcar con una "X" su respuesta sobre el número que más refleja la manera en que usted percibe el desempeño e innovación en su institución en los últimos tres años como resultado de la gestión de sus recursos humanos. Si no está absolu- tamente seguro sobre su respuesta, por favor, elija aquella opción que más se aproxime a la situación que percibe en su institución. Su opinión es valiosa.

Para esta sección la escala de valoración es la siguiente:

\begin{tabular}{lll}
\hline $1=$ Pésimo & $2=$ Malo & $3=$ Regular \\
\hline $4=$ Bueno & $5=$ Superior \\
\hline
\end{tabular}

\begin{tabular}{lccccc}
\hline Cómo evalúa el desempeño de su institución en los últimos tres años & & & & \\
\hline D1. De la imagen de su institución y servicios & 1 & 2 & 3 & 4 & 5 \\
\hline D2. De la calidad académica de los procesos y servicios educativos proporcionados & 1 & 2 & 3 & 4 & 5 \\
\hline D3. De la formación de calidad de los estudiantes & 1 & 2 & 3 & 4 & 5 \\
\hline D4. Del valor añadido a los procesos-servicios otorgados & 1 & 2 & 3 & 4 & 5 \\
\hline D5. De la satisfacción de los usuarios con los procesos o servicios proporcionados & 1 & 2 & 3 & 4 & 5 \\
\hline D6. Del desempeño en el logro de los objetivos e indicadores académicos alcanzados & 1 & 2 & 3 & 4 & 5 \\
\hline D7. De la calidad en los procesos-servicios proporcionados & 1 & 2 & 3 & 4 & 5 \\
\hline D8. Del nivel de tecnología incorporado en los procesos-servicios educativos & 1 & 2 & 3 & 4 & 5 \\
\hline D9. De la productividad de nuestros empleados & 1 & 2 & 3 & 4 & 5 \\
\hline D10. De la eficiencia en los procesos y actividades de gestión & 1 & 2 & 3 & 4 & 5 \\
\hline D11. Del desempeño en la aplicación de los recursos & 1 & 2 & 3 & 4 & 5 \\
\hline D12. De la mejora en la coordinación de los procesos internos & 1 & 2 & 3 & 4 & 5 \\
\hline D13. De la innovación continua en los procesos-servicios educativos & 1 & 2 & 3 & 4 & 5 \\
\hline
\end{tabular}

SECCION TRES. Instrucciones: Para mejorar los resultados académicos en su institución, ¿qué recomienda usted que se deba diseñar e implementar en la gestión del capital humano (competencias, experiencia, habilidad, talento del personal, entre otros)?

SECCION CUATRO. Datos generales del encuestado y de su institución

\begin{tabular}{|c|}
\hline 1. Puesto/cargo: Director_____Subdirector_____ Jefe de área______ Docente______ Administrativo _______ \\
\hline $\begin{array}{l}\text { 2. Antigüedad en el puesto: De } 0 \text { a } 5 \text { años____ De } 6 \text { a } 10 \text { años_____ De } 11 \text { a } 15 \text { años______ De } 16 \text { a } 20 \text { años____ De } 21 \text { a } \\
24 \text { años____ De } 25 \text { a } 30 \text { años_____ Más de } 31 \text { años_____ }\end{array}$ \\
\hline $\begin{array}{l}\text { 3. Edad: De } 18 \text { a } 25 \text { años_____ De } 26 \text { a } 33 \text { años_____De } 34 \text { a } 41 \text { años_____ De } 42 \text { a } 49 \text { años_____ De } 50 \text { años en } \\
\text { adelante___- }\end{array}$ \\
\hline 4. Escolaridad: Primaria___Secundaria____Preparatoria_____Licenciatura____Posgrado____ \\
\hline 6. CBTIS 271___ 236 \\
\hline 7.Zona: Norte________Centro_________Sur________ \\
\hline
\end{tabular}

